\title{
Optimal Synthesis of Four-Bar Linkage Path Generation through Evolutionary Computation with a Novel Constraint Handling Technique
}

\author{
Suwin Sleesongsom $\mathbb{D}^{1}$ and Sujin Bureerat $\mathbb{D}^{2}$ \\ ${ }^{1}$ Department of Aeronautical Engineering, International Academy of Aviation Industry, \\ King Mongkut's Institute of Technology Ladkrabang, Bangkok 10520, Thailand \\ ${ }^{2}$ Sustainable and Infrastructure Research and Development Center, Department of Mechanical Engineering, \\ Faculty of Engineering, Khon Kaen University, Khon Kaen 40002, Thailand \\ Correspondence should be addressed to Suwin Sleesongsom; suwin.se@kmitl.ac.th
}

Received 26 March 2018; Revised 20 May 2018; Accepted 19 September 2018; Published 1 November 2018

Academic Editor: Saeid Sanei

Copyright ( 12018 Suwin Sleesongsom and Sujin Bureerat. This is an open access article distributed under the Creative Commons Attribution License, which permits unrestricted use, distribution, and reproduction in any medium, provided the original work is properly cited.

\begin{abstract}
This paper presents a novel constraint handling technique for optimum path generation of four-bar linkages using evolutionary algorithms (EAs). Usually, the design problem is assigned to minimize the error between desired and obtained coupler curves with penalty constraints. It is found that the currently used constraint handling technique is rather inefficient. In this work, we propose a new technique, termed a path repairing technique, to deal with the constraints for both input crank rotation and Grashof criterion. Three traditional path generation test problems are used to test the proposed technique. Metaheuristic algorithms, namely, artificial bee colony optimization (ABC), adaptive differential evolution with optional external archive (JADE), population-based incremental learning (PBIL), teaching-learning-based optimization (TLBO), real-code ant colony optimization (ACOR), a grey wolf optimizer (GWO), and a sine cosine algorithm (SCA), are applied for finding the optimum solutions. The results show that new technique is a superior constraint handling technique while TLBO is the best method for synthesizing fourbar linkages.
\end{abstract}

\section{Introduction}

Since the last decade, many researchers have tried to solve the optimization for path generation of four-bar linkages using metaheuristic (MH) algorithms. The objective of path generation problem is to find dimensions of a mechanism, which minimize the target path and the actual path of a point on the coupler link. Path synthesis is one type of kinematic syntheses of four-bar mechanisms [1-13] in which such syntheses are basically classified into two groups. The first category is called dimensional synthesis [1, 2, 4, 5, 7-10]. This synthesis type aimed to find significant link lengths to achieve desirable function, path, and motion generation. The second synthesis is called type synthesis $[6,11]$ where a designer initially specifies a predefined motion transmission and is supposed not initially to know the mechanism type.
This method is analogous to topology design in structural optimization. Having finished synthesizing, a certain mechanism type is received. Position analysis of the four-bar mechanism can be categorized into two groups. The first one is a vector loop or loop closure equation, which is the most traditional method in kinematic analysis, and it is one of the most popular analyses for path synthesis [1, 2, 4, 5, 7-15]. This equation can be solved by using Freudenstein equation. The second analysis technique is a straight forward and a simple method for position analysis involving the use of trigonometric laws for triangles, e.g., the law of cosine $[3,16,17]$, whereas the six-bar linkage for steering mechanism also uses the same technique [18]. This work proposes a new computing technique for four-bar linkage position analysis by employing the concept of drawing an arbitrary rectangle using two circles. 
The mechanism synthesis can be converted into optimization problem and be solved by using optimizers, where both nongradient- and gradient-based algorithms have been solved this problem. Recently, a nongradient-based optimizer, e.g., evolutionary algorithms (EAs) or metaheuristics (MHs), is a more popular selection in solving such optimization problems. It has been found that the advantages of using MHs are robustness, simplicity of use, and independence of function derivatives; however, they unavoidably lack convergence speed and consistency. At present, many algorithms in this group have been developed, which are expected to enhance in both convergence speed and consistency. Some of the most frequently used MHs for path synthesis are differential evolution (DE) $[2,3,5,8,9,11,13]$, genetic algorithms (GA) [5, 13], particle swarm optimization (PSO) $[5,13]$, and an imperialist competitive algorithm (ICA) [13], etc. The use of gradient-based method, on the other hand, is somewhat questionable to deal with global optimization and nonsmooth constraints in the path synthesis. Nevertheless, if those aforementioned factors can be alleviated, the advantages of the gradient-based method are better convergence rate and consistency. In the literature, many researchers have combined $\mathrm{MHs}$ and a gradient-based optimizer for solving many kinds of real world problems, which is called a hybrid algorithm. Especially for part synthesis, the hybrid optimizers are introduced as the ant gradient [1], hybrid GA [4], and hybrid GA with sequential quadratic programming (SQP) [12]. The hybridization between two or more MHs was also studied such as hybrid GA-DE [7]. Furthermore, the path synthesis is extended to multiobjective optimization, which was solved by using a multiobjective genetic algorithm (MOGA) [10]. From the review literature, it was found that some MHs have been used for solving this task except the work by Sleesongsom and Bureerat [17]; therefore, one of the objectives of this paper is to present the comparative performance of a number of currently used MHs. Those algorithms include the artificial bee colony optimization (ABC), adaptive differential evolution with optional external archive (JADE), population-based incremental learning (PBIL), teaching-learning-based optimization (TLBO), the realcode ant colony optimization (ACOR), a grey wolf optimizer (GWO), a Jaya algorithm (Jaya), and a sine cosine algorithm (SCA).

The path generation is a mechanism synthesis to make a point on a coupler link move along the target path; thus, the objective function is the minimization of the sum square error between the target path and the actual path [5]. The design problem is a constrained optimization problem that comprises two constraints. The first constraint is set for the shortest link in the mechanism to be able to rotate with a complete revolution (crank) in either direction (clockwise or counter clockwise). The second constraint is assigned such that the four link lengths satisfy the Grashof criterion which results in a crank-rocker. From previous work, a simple exterior penalty function technique had used to deal with these constraints $[1-5,7,8,10-13]$. The new technique proposed by [17] to neglect the first constraint from the optimization problem, which found new technique, provided the better result than the traditional exterior penalty technique. Additionally, the technique had improved in the result, but it increased in time consuming. From the present study can be concluded that the constraint handling technique is an inefficient technique, which needs an improvement [9, 13, 14]. Phukaokaew et al. [14] studied the number of unsuccessful runs from performing $\mathrm{MHs}$ for 30 runs, where the path synthesis optimization problems employ the penalty function (PF) technique. This means there is no guarantee that using this technique can promote the good results. The reason is that using the penalty technique leads to an overly narrow feasible region. As a consequence, MHs, which mostly have slow convergence rates, struggle to reach an optimum. Ebrahimi and Payvandy proposed the way to improve the constraint handling technique, which was still based on a penalty function, and they believe the proposed method can enhance the search performance [13].

This paper focuses on two aspects of investigation. Firstly, a new constraint handling technique for path synthesis of a four-bar linkage using MHs is proposed. The method is based on repairing illegitimate design solutions to become feasible solutions. The second investigation is to study the performance comparison of a number of established MHs for solving four-bar linkage path synthesis with the new constraint handling technique, where both convergence rate and consistency of the methods are measured.

The rest of this paper is organized as follows. Section 2 proposes an alternative position analysis of a four-bar linkage. The optimization problem and the constraint handling technique are detailed in Section 3. The numerical experiments are given in Section 4, while the design results are discussed in Section 5. The discussions and conclusions of the study are finally drawn in Sections 6 and 7, respectively.

\section{Position Analysis of Four-Bar Linkages}

The kinematic diagram of a four-bar linkage is shown in Figure 1. The four-bar linkage is the simplest and most commonly used linkage in many engineering applications. It is composed of a kinematic chain of four binary links connected with four revolute joints (denoted by capital letters) with one link being assigned as a frame. Applications for this mechanism are a window wiper, a door closing mechanism, rock crushers, etc. [9]. Based on the Gruebler equation for planar mechanisms, the mobility or degree-offreedom of the mechanism is one; thus, it is a constrained mechanism fully operated by one actuator. The path generation for a four-bar linkage is a dimension-based design of the four-bar linkage lengths $\left(r_{1}, r_{2}, r_{3}, r_{4}\right)$ and other parameters, which makes the trace point $\left(x_{P}, y_{P}\right)$ on the coupler link follow the desire path $\left(x_{d}, y_{d}\right)$.

Let the coordinates of the joints $\mathrm{O}_{2}$ and $\mathrm{O}_{4}$ be $\left(x_{2}, y_{2}\right)$ and $\left(x_{4}, y_{4}\right)$, respectively, so the coordinates of points $B$ and $\mathrm{O}_{4}$ can be computed as 




Figure 1: Four-bar linkage in a global coordinate system.

$$
\begin{aligned}
& x_{B}=x_{2}+r_{2} \cos \left(\theta_{2}\right), \\
& y_{B}=y_{2}+r_{2} \sin \left(\theta_{2}\right), \\
& x_{4}=x_{2}+r_{1} \cos \left(\theta_{0}\right), \\
& y_{4}=y_{2}+r_{1} \sin \left(\theta_{0}\right),
\end{aligned}
$$

where the angular positions $\theta_{0}$ and $\theta_{2}$ are positive counterclockwise. The positions of points $C$ and $C^{\prime}$ are the intersection points of two circles as illustrated in Figure 1, which is calculated by a vector approach. First, let $B$ and $\mathrm{O}_{4}$ be the centres of the circles with radii $r_{3}$ and $r_{4}$, respectively, and let $d$ be the distance between $B$ and $O_{4}$. Then, generate $\mathbf{V}_{1}$, the unit vector from $B$ to $O_{4}$, and $\mathbf{V}_{2}$, the unit vector perpendicular to $\mathbf{V}_{1}$. Given that $\mathbf{V}_{3}$ is the vector from $B$ to one of the intersection points, the angle between $\mathbf{V}_{1}$ and $\mathbf{V}_{3}$ denoted by $A$ is solved using the law of cosines

$$
r_{3}^{2}=r_{4}^{2}-d^{2}+2 r_{3} d \cos (A)
$$

The intersection points can then be obtained as

$$
\begin{aligned}
\mathbf{r}_{C} & =\mathbf{r}_{B}+\left(r_{3} \cos A\right) \mathbf{V}_{1}+\left(r_{3} \sin A\right) \mathbf{V}_{2}, \\
\mathbf{r}_{C^{\prime}} & =\mathbf{r}_{B}+\left(r_{3} \cos A\right) \mathbf{V}_{1}-\left(r_{3} \sin A\right) \mathbf{V}_{2} .
\end{aligned}
$$

The coupler curve is formed when the crank link rotates. From Figure 1, the coupler point coordinates $\left(\mathbf{r}_{P}\right)$ in the global coordinate can be expressed as

$$
\mathbf{r}_{P}=\mathbf{r}_{B}+\mathbf{r}_{P / B}=\mathbf{r}_{B}+\mathbf{r}_{P x}+\mathbf{r}_{P y}=\mathbf{r}_{B}+\mathbf{r}_{P x} \mathbf{e}_{P x}+\mathbf{r}_{P y} \mathbf{e}_{P y},
$$

where $\mathbf{e}_{P x}$ is a unit vector in the direction from $B$ to $C$ and $\mathbf{e}_{P y}$ is a unit vector perpendicular to $\mathbf{e}_{P x}$. The distances $\mathbf{r}_{P x}$ and $\mathbf{r}_{P y}$ can be computed using the given dimensions of $r_{3}$, $P B$, and $P C$. Also, they can be set as design variables for optimal path synthesis. These vector forms of position analysis are used for four-bar linkage synthesis in this paper.

\section{Optimization Problem and Constraint Handling}

3.1. Optimization Problem. The path synthesis problem is converted to an optimization problem with an objective function expressed as the sum of square errors between the distances of $\mathbf{r}_{d}$ and their corresponding $\mathbf{r}_{P}$. A set of the input angles $\left(\theta_{2}\right)$ is assigned as design variables if the prescribed timing is not given. There are two major constraints. The first constraint is set in such a way that the generated mechanism type is a crank-rocker. This leads to two constraints: (i) sum of the shortest and longest links of linkage must be less than the sum of two remaining links and (ii) the shortest link must be an input link where one of its nearby links is set as a frame. The constraint (i) is denoted with Equation (7), while the constraint (ii) is denoted with Equation (6). In cases where the prescribed timing is not promoted, the second constraint is that the input values of $\theta_{2}$ must be in 
either ascending or descending order. The constraint (ii) is added to the design problem, which is denoted by $\theta_{2}^{i}$, where $i=1$. The optimization problem can then be written as

$$
\min f_{\mathrm{obj}}(\mathbf{x})=\sum_{i=1}^{N}\left[\left(x_{d, i}-x_{P, i}\right)^{2}+\left(y_{d, i}-y_{P, i}\right)^{2}\right] \text {, }
$$

subject to

$$
\begin{aligned}
& \min \left(r_{1}, r_{2}, r_{3}, r_{4}\right)=\text { crank, } \\
& 2 \min \left(r_{1}, r_{2}, r_{3}, r_{4}\right)+2 \max \left(r_{1}, r_{2}, r_{3}, r_{4}\right) \\
& \quad<\left(r_{1}+r_{2}+r_{3}+r_{4}\right), \\
& \theta_{2}^{i}<\theta_{2}^{i+1}<\cdots<\theta_{2}^{N}, \quad i=1, \\
& \mathbf{x}_{l} \leq \mathbf{x} \leq \mathbf{x}_{u},
\end{aligned}
$$

where $\mathbf{x}=\left\{r_{1}, r_{2}, r_{3}, r_{4}, \mathbf{r}_{P x}, \mathbf{r}_{P y}, \theta_{0}, x_{\mathrm{O}_{2}}, y_{\mathrm{O}_{2}}, \theta_{2}^{i}\right\}^{T}, N$ is the number of points on prescribed or target curve, and $\theta_{2}^{i}$ are input link angles. $\mathbf{x}_{l}$ and $\mathbf{x}_{u}$ are lower and upper bounds of a design vector $\mathbf{x}$, respectively.

A function evaluation is carried out for the optimization problem (5) by implementing the position analysis detailed in Section 2. To enable the position analysis, the constraints (6)-(8) must be first satisfied. In the previous studies, if the conditions are not met, the objective function will be modified by adding to it a great penalty value. However, in this work, the proposed path repairing algorithm (PRA) is assigned to repair all design solutions to always be feasible before performing position analysis of a four-bar linkage.

3.2. Constraint Handling. Normally, a traditional exterior penalty function technique has been used with the constrained optimization problem (5) $[1-5,7,8,10-13]$, but it was found to be inefficient $[9,13,14,17]$. Using such a technique is questionable when prespecifying a penalty parameter. If the parameter is too small, the resulting optimum solution may be infeasible, but if it is too large, $\mathrm{MH}$ may not be able to find the optimum. This leads us to propose a new strategy to deal with the constraints called the path repairing technique. The technique can be separated into two parts as repair of the input link angle constraint (8) and the Grashof criterion constraint Equations (6) and (7).

3.2.1. Input Link Angle Constraint. For an optimization without prescribed timing, a repairing technique for this phase is shown in Algorithm 1 where the input variables are $\mathbf{x}=\left\{r_{1}, r_{2}, r_{3}, r_{4}, \mathbf{r}_{P x}, \mathbf{r}_{P y}, \theta_{0}, x_{O_{2}}, y_{O_{2}}, \theta_{2}^{i}\right\}^{T}$. Once it is found that the values of $\theta_{2}^{i}$ are not in either ascending or descending orders, the design variables $\mathbf{x}$ in the part of $\theta_{2}^{i}$ will be repaired. Firstly, $N-1$ uniform random number $\alpha_{i} \in[0.0001,1]$ for $i=1, \ldots, N-1$ is generated. The lower bound is set to be 0.0001 in order to avoid repeated values of $\theta_{2}^{i}$ in cases that some random numbers $\alpha_{i}$ become zeros. Those random values are then scaled in step 2 so that the sum of them does not exceed $2 \pi$. The first angular position of an input link is $\theta_{2}^{1}$, its original value. Then, the next value of $\alpha_{i}$ for $i=1, \ldots, N-1$ is accumulatively added to the next input angle until the last value is obtained as $\theta_{2}^{N}=\theta_{2}^{1}+\alpha_{1}+\cdots+\alpha_{N-1}$. Then, the new set of input angles is in an ascending direction before returning to the position analysis of a four-bar linkage. This concept was successfully used in a sprayed plate fin heat sink design [19]. The obtained sequence of input angles always obeys the constraint (8).

The vector of design variables for path synthesis of a particular four-bar linkage is $\mathbf{x}=\left\{r_{1}, r_{2}, r_{3}, r_{4}, \mathbf{r}_{P x}, \mathbf{r}_{P y}\right.$, $\left.\theta_{0}, x_{2}, y_{2}, \theta_{2}^{1}, \theta_{2}^{2}, \theta_{2}^{3}, \theta_{2}^{4}, \theta_{2}^{5}, \theta_{2}^{6}\right\}^{T}$. Variables $r_{1}-\mathbf{r}_{4} \in[5,60]$ are the link lengths of the linkage, and $\theta_{2}^{1}-\theta_{2}^{6} \in[0,2 \pi]$ are the angular positions of link $r_{2}$, also known as timings of the crank, while other variables are shown in Figure 1. The legitimated set of the timings must obey the condition $\theta_{2}^{1}<\theta_{2}^{2}<\theta_{2}^{3}<\theta_{2}^{4}<\theta_{2}^{5}<\theta_{2}^{6}$. During an optimization process, if the set of timings is decoded as, for example, $\theta_{2}^{1}=0.5, \theta_{2}^{2}=$ $0.45, \theta_{2}^{3}=0.67, \theta_{2}^{4}=1.35, \theta_{2}^{5}=4.50$, and $\theta_{2}^{6}=2.10$, they violate constraint (8). Then, Algorithm 1 is activated to repair these values. Five (for 6 timings) uniform random numbers are generated, for example, $\alpha_{1}=0.5, \alpha_{2}=0.15, \alpha_{3}=0.75$, $\alpha_{4}=0.45$, and $\alpha_{5}=0.30$. The values of $\alpha_{i}$ are then scaled down according to step 2 in Algorithm 1 to meet the condition $\theta_{2}^{1}-\theta_{2}^{6} \in[0,2 \pi]$ leading to

$$
\begin{aligned}
& \alpha_{1}=1.99 \pi \times \frac{0.5}{(6-1)}=0.6252, \\
& \alpha_{2}=1.99 \pi \times \frac{0.15}{(6-1)}=0.1876, \\
& \alpha_{3}=1.99 \pi \times \frac{0.75}{(6-1)}=0.9378, \\
& \alpha_{4}=1.99 \pi \times \frac{0.45}{(6-1)}=0.5627, \\
& \alpha_{5}=1.99 \pi \times \frac{0.3}{(6-1)}=0.3751 .
\end{aligned}
$$

The output modified values of $\theta_{2}^{1}-\theta_{2}^{6}$ are then computed as

$$
\begin{aligned}
& \theta_{2}^{1}=\theta_{2}^{1}=0.5 \\
& \theta_{2}^{2}=\theta_{2}^{1}+\alpha_{1}=1.1252 \\
& \theta_{2}^{3}=\theta_{2}^{1}+\alpha_{1}+\alpha_{2}=1.3128 \\
& \theta_{2}^{4}=\theta_{2}^{1}+\alpha_{1}+\alpha_{2}+\alpha_{3}=2.2506 \\
& \theta_{2}^{5}=\theta_{2}^{1}+\alpha_{1}+\alpha_{2}+\alpha_{3}+\alpha_{4}=2.8133 \\
& \theta_{2}^{6}=\theta_{2}^{1}+\alpha_{1}+\alpha_{2}+\alpha_{3}+\alpha_{4}+\alpha_{5}=3.1884
\end{aligned}
$$

As a result, by using Algorithm 1, the timings are always feasible. The difference between $\theta_{2}^{6}$ and $\theta_{2}^{1}$ will never exceed $2 \pi$.

3.2.2. Grashof's Criterion Constraint. With the same reasons as for the previous constraint, the dimensions of $\left\{r_{1}, r_{2}, r_{3}, r_{4}\right\}$ must obey the conditions (6) and (7) so that the resulting mechanism is usable. Let the bound constraints of $\left\{r_{i}\right\}$ in Equation (9) be separately defined as 
Input variables: infeasible $\theta_{2}^{i}, \theta_{2}^{i+1}, \ldots, \theta_{2}^{N}$

Output variables: feasible $\theta_{2}^{i}, \theta_{2}^{i+1}, \ldots, \theta_{2}^{N}$

(1) Generate a uniform random number $\left(\alpha_{1}+\cdots+\alpha_{N-1}\right)$ whose values are in the range of $[0.0001,1]$

(2) Set $\alpha_{i}=1.99 \pi /(N-1) \alpha_{i}$, for $i=1, \ldots, N-1$.

(3) For $i=1$ to $N-1$

(4) Generate $\theta_{2}^{i+1}=\theta_{2}^{i}+\alpha_{i-1}$

(5) End

Algorithm 1: A repairing technique for the prescribed timing constraint.

$\left\{r_{1, l}, r_{2, l}, r_{3, l}, r_{4, l}\right\} \leq\left\{r_{1}, r_{2}, r_{3}, r_{4}\right\} \leq\left\{r_{1, u}, r_{2, u}, r_{3, u}, r_{4, u}\right\}$.

Then, the relation can be found:

$$
\begin{aligned}
r_{\min } & =\max \left\{r_{1, l}, r_{2, l}, r_{3, l}, r_{4, l}\right\} \leq\left\{r_{1}, r_{2}, r_{3}, r_{4}\right\} \\
& \leq \min \left\{r_{1, u}, r_{2, u}, r_{3, u}, r_{4, u}\right\}=r_{\max } .
\end{aligned}
$$

The Grashof criterion repairing technique is shown in Algorithm 2. Firstly, four uniform random numbers $\left\{\delta_{1}, \delta_{2}, \delta_{3}, \delta_{4}\right\}$ in the range of $[0.0001,1]$ are generated. The lower bound is set to be 0.0001 for the same reason as Algorithm 1. Then the auxiliary variables $S_{i}$ for $i=1, \ldots, 4$ are computed as

$$
\begin{aligned}
& S_{2}=\delta_{2}, \\
& S_{3}=\delta_{2}+\delta_{3}, \\
& S_{4}=\delta_{2}+\delta_{3}+\delta_{4}, \\
& S_{1}=\delta_{1}+\delta_{2}+\delta_{3}+\delta_{4} .
\end{aligned}
$$

With this computation, it is concluded that $S_{2}$ is their minimum and $S_{1}$ is their maximum. These four values fulfil the Grashof criterion if $S_{2}$ is an input crank. Condition (7) also holds if

$$
\begin{gathered}
S_{1}+S_{2}<S_{3}+S_{4}, \\
\delta_{1}+2 \delta_{2}+\delta_{3}+\delta_{4}<2 \delta_{2}+2 \delta_{3}+\delta_{4}, \\
\text { or } \delta_{1}<\delta_{3} .
\end{gathered}
$$

Therefore, the computational steps 3-4 in Algorithm 2 are applied. Then, the values of $\left\{S_{i}\right\}$ are all scaled down so that $\max \left(S_{i}\right)=S_{1} \leq 1$. The values of $b_{i}$ can then be computed as

$$
b_{i}=r_{\min }+\left(r_{\max }-r_{\min }\right) S_{i,} \text { for } i=1, \ldots, 4 .
$$

Then, set $r_{2}=b_{2}$. The values of $b_{1}, b_{3}$, and $b_{4}$ are assigned to be the lengths of $r_{1}, r_{3}$ and $r_{4}$, respectively. With such a computing scheme, the values of $\left\{r_{1}, r_{2}, r_{3}, r_{4}\right\}$ returned from activating Algorithm 2 will always be feasible. In the search process of $\mathrm{MH}$, when a function evaluation is revoked, feasibility of a design solution $\mathbf{x}$ will be checked. If it is infeasible, Algorithms 1 and 2 will be used to repair or alter the values of $\theta_{2}^{i}$ and $r_{i}$ in $\mathbf{x}$ and send them back to the main process of a metaheuristic. That means all design solutions in a population of $\mathrm{MH}$ are always feasible.

Given that, for example, $r_{1}=15, r_{2}=10, r_{3}=30$, and $r_{4}=20$, the Grashof criterion is not fulfilled since $r_{2}+r_{3}>r_{1}+r_{4}$. These values will then be regenerated by using Algorithm 2. From step 1, given that the values of $\delta_{1}-\delta_{4}$ are randomly generated as $\delta_{1}=0.01, \delta_{2}=0.55$, $\delta_{3}=0.25$, and $\delta_{4}=0.45$, then the auxiliary variables are computed based on (14) as

$$
\begin{aligned}
& S_{1}=0.01+0.55+0.25+0.45=1.26 \\
& S_{2}=0.55=0.55 \\
& S_{3}=0.55+0.25=0.80 \\
& S_{4}=0.55+0.25+0.45=1.25
\end{aligned}
$$

Since $S_{1}$ is greater than 1.00 , the values of the auxiliary variables are modified as

$$
\begin{aligned}
& S_{1}=\frac{1.26}{1.26}=1.0000, \\
& S_{2}=\frac{0.55}{1.26}=0.4365, \\
& S_{2}=\frac{0.80}{1.26}=0.6349, \\
& S_{4}=\frac{1.25}{1.26}=0.9921 .
\end{aligned}
$$

Thus, the new link length values are obtained using (16):

$$
\begin{aligned}
& r_{1}=5+(60-5) \times 1.0000=60.0000, \\
& r_{2}=5+(60-5) \times 0.4365=29.0079, \\
& r_{3}=5+(60-5) \times 0.6349=39.9195, \\
& r_{4}=5+(60-5) \times 0.9921=59.5655,
\end{aligned}
$$

which results in a crank-rocker four-bar linkage. In cases where the generated value of $\delta_{1}$ is greater than that of $\delta_{3}$, their values are swapped. If they are equal, Step 3 in Algorithm 2 is activated ensuring that a crack-rocker is obtained after the repairing process.

\section{Numerical Experiment}

For evaluating the performance of the proposed path repairing technique, three path synthesis test problems of a four-bar linkage are used, whereas the optimizers are 7 established MHs. To validate the new approach, it will be compared with the exterior penalty function technique, which is traditionally applied in previous work. The path generation problems are detailed as $[5,14]$ : 
Input: infeasible $\left\{r_{1}, r_{2}, r_{3}, r_{4}\right\}$ and bounds: $r_{\min }, r_{\max }$

Output: feasible $\left\{r_{1}, r_{2}, r_{3}, r_{4}\right\}$

(1) Generate uniform random numbers $\left\{\delta_{1}, \delta_{2}, \delta_{3}, \delta_{4}\right\}$ whose values are in the range of $[0.0001,1]$

(2) If $\delta_{1}>\delta_{3}$, swop their positions

(3) Else if $\delta_{1}=\delta_{3}$, set $\delta_{3}=\delta_{3}+0.001$

(4) Assign values

$S_{2}=\delta_{2}$

$S_{3}=\delta_{2}+\delta_{3}$

$S_{4}=\delta_{2}+\delta_{3}+\delta_{4}$

$S_{1}=\delta_{1}+\delta_{2}+\delta_{3}+\delta_{4}$

(5) If $\max \left(S_{1}, S_{2}, S_{3}, S_{4}\right)>1$, compute $\left\{S_{1}, S_{2}, S_{3}, S_{4}\right\}=\left\{S_{1}, S_{2}, S_{3}, S_{4}\right\} / \max \left(S_{1}, S_{2}, S_{3}, S_{4}\right)$

(6) Compute $b_{i}=r_{\min }+\left(r_{\max }-r_{\min }\right) S_{i}$ for $i=1, \ldots, 4$

(7) Assign $r_{2}=b_{2}$ and assign $b_{1}, b_{3}$, and $b_{4}$ as the lengths of $r_{1}, r_{3}$, and $r_{4}$, respectively

Algorithm 2: Repairing Grashof criterion constraint.

Case 1. Path generation without prescribed timing

Design variables are

$\mathbf{x}=\left\{r_{1}, r_{2}, r_{3}, r_{4}, \mathbf{r}_{P x}, \mathbf{r}_{P y}, \theta_{0}, x_{2}, y_{2}, \theta_{2}^{1}, \theta_{2}^{2}, \theta_{2}^{3}, \theta_{2}^{4}, \theta_{2}^{5}, \theta_{2}^{6}\right\}^{T}$.

Target points are $r_{d}^{i}=\{(20,20),(20,25),(20,30),(20$, $35),(20,40),(20,45)\}$.

Limits of the design variables are as follows:

$$
\begin{aligned}
5 & \leq r_{1}, r_{2}, r_{3}, r_{4} \leq 60, \\
-60 & \leq \mathbf{r}_{P x}, \mathbf{r}_{P y}, x_{2}, y_{2} \leq 60, \\
0 & \leq \theta_{0}, \theta_{2}^{1}, \theta_{2}^{2}, \theta_{2}^{3}, \theta_{2}^{4}, \theta_{2}^{5}, \theta_{2}^{6} \leq 2 \pi .
\end{aligned}
$$

Case 2. Path generation with prescribed timing

Design variables are

$$
\begin{aligned}
\mathbf{x} & =\left\{r_{1}, r_{2}, r_{3}, r_{4}, \mathbf{r}_{P x}, \mathbf{r}_{P y}, \theta_{0}, x_{2}, y_{2}\right\}^{T}, \\
\theta_{2}^{i} & =\left[\frac{\pi}{6}, \frac{\pi}{3}, \frac{\pi}{2}, \frac{2 \pi}{3}, \frac{5 \pi}{6}, \pi\right] .
\end{aligned}
$$

Target points are $r_{d}^{i}=\{(0,0),(1.9098,5.8779),(6.9098$, 9.5106), (13.09, 9.5106), (18.09, 5.8779), $(20,0)\}$.

Limits of the design variables are

$$
\begin{aligned}
5 & \leq r_{1}, r_{2}, r_{3}, r_{4} \\
-50 & \leq \mathbf{r}_{P x}, \mathbf{r}_{P y}, x_{2}, y_{2} \\
0 & \leq \theta_{0}
\end{aligned}
$$

Case 3. Path generation without prescribed timing Design variables are

$$
\begin{aligned}
\mathbf{x}= & \left\{r_{1}, r_{2}, r_{3}, r_{4}, \mathbf{r}_{P x}, \mathbf{r}_{P y}, \theta_{0}, x_{2}, y_{2}, \theta_{2}^{1}, \theta_{2}^{2}, \theta_{2}^{3}, \theta_{2}^{4}, \theta_{2}^{5}, \theta_{2}^{6}, \theta_{2}^{7},\right. \\
& \left.\theta_{2}^{8}, \theta_{2}^{9}, \theta_{2}^{10}\right\}^{T} .
\end{aligned}
$$

Target points are $r_{d}^{i}=\{(20,10),(17.66,15.142),(11.736$, 17.878), (5, 16.928), (0.60307, 12.736), (0.60307, 7.2638), (5, $3.0718),(11.736,2.1215),(17.66,4.8577),(20,10)\}$.

Limits of the design variables are

$$
\begin{aligned}
5 & \leq r_{1}, r_{2}, r_{3}, r_{4} \leq 80, \\
-80 & \leq \mathbf{r}_{P x}, \mathbf{r}_{P y}, x_{2}, y_{2} \leq 80, \\
0 & \leq \theta_{0}, \theta_{2}^{1}, \theta_{2}^{2}, \theta_{2}^{3}, \theta_{2}^{4}, \theta_{2}^{5}, \theta_{2}^{6}, \theta_{2}^{7}, \theta_{2}^{8}, \theta_{2}^{9}, \theta_{2}^{10} \leq 2 \pi .
\end{aligned}
$$

The first synthesis problem has a straight line target path without a prescribed timing. The second test problem has a circular prescribed path with prescribed timing while the third test problem has an elliptic path without prescribed timing. The optimizers used to tackle the test problems are 7 well-known and newly developed metaheuristics. Their optimization parameter settings are given below. The population size $n_{P}=100$ is used for all algorithms, unless otherwise specified. It should be noted that the terms and variable definitions are from their original sources.

(1) Artificial bee colony algorithm (ABC) [20]: the number of food sources for employed bees is set to be $n_{P} / 2$. A trial counter to discard a food source is 100 .

(2) Real-code ant colony optimization (ACOR) [21]: the parameter settings are $q=0.2$ and $\xi=1$.

(3) Teaching-learning-based optimization (TLBO) [22]: parameter settings are not required.

(4) Adaptive differential evolution with optional external archive (JADE) [23]: The parameters are selfadapted during an optimization process.

(5) Population-based incremental learning (PBIL) [24]. The learning rate, mutation shift, and mutation rate are set as $0.5,0.7$, and 0.2 , respectively.

(6) Grey wolf optimizer (GWO) [25]: the GWO has only two main parameters to be adjusted, $a$ and $C$, where $a$ is decreased from 2 to 0 and $C$ is randomly generated in the range of $[0,2]$.

(7) Sine cosine algorithm (SCA) [26]. The parameter $r_{1}$ decreases linearly from $a=2$ to 0 . 
It should be noted that the parameter settings used in this paper are either the default values provided or suggested by their authors. Also, they have been used in some previous studies with acceptable results [17, 27-30].

The total number of iterations or generations for each optimization run is set to be 500 . Any $\mathrm{MH}$ that uses a different population size will be terminated with the total number of function evaluations as $100 \times 500$. Each $\mathrm{MH}$ runs 30 times, so that to measure its convergence rate and consistency. It should be noted that most works in the literature tended to ignore examining the search consistency of MHs. This can be carried out by running MHs many times. In this paper, each $\mathrm{MH}$ is run 30 times for each optimization problem. The means of the objective function values obtained from the various MHs are used as their performance indicator. Also, the comparison based on nonparametric statistical Friedman test $[17,31]$ is employed. Thus, this study would be a proper baseline for the topic of using MHs for four-bar linkage path synthesis in the future.

In conclusion, the path repairing technique is proposed to increase the performance in solving four-bar linkage path synthesis. To evaluate the performance of the proposed technique, three path synthesis test problems and 7 established MHs are used to study. To validate the new constraint handling approach, it is compared with the results that obtained from using the classical exterior penalty function technique, the most popularly used technique for path synthesis. Moreover, statistical results including mean, minimum, maximum, and standard deviation are also reported.

\section{Design Results}

The optimization of path generation of four-bar mechanisms is to find the optimized link lengths and some other parameters, which minimize the objective function. Three case studies with and without prescribed timing are considered for performance testing of the optimizers and the new constraint handling technique. The mean values of objective functions are used as the main performance indicator, where the lower mean value is more reliable optimizer. The mean objective function value determines $\mathrm{MH}$ reliability as $\mathrm{MH}$ with lower mean value is likely to be more successful in solving the problem, even with only one optimization run.

The results of Case 1 obtained from the seven optimizers with the novel path repairing technique and the penalty technique are shown in Tables 1 and 2 respectively. In the tables, the number of successful runs (a run that obtains a feasible solution), the mean objective function values from 30 optimization runs (Mean), the worse result (Max), the best result (Min), the standard deviation (Std), and the best linkage that gives the minimum objective function value of each algorithm are given. Each parameter computes based on the objective function defined as the sum of squares of the distances between the desired points, and the actual points, while the term "Error" in the tables is an average distance error of the best found solution computed as

$$
\text { Error }=\frac{1}{N} \sum_{i=1}^{N} \sqrt{\left(x_{d, i}-x_{P, i}\right)^{2}+\left(y_{d, i}-y_{P, i}\right)^{2}} \text {, }
$$

which has been used as a performance indicator in some previously published papers. $N$ is the number of points on the prescribed or target curve. From the results, it is seen that the proposed path repairing technique is far superior to the exterior penalty technique used in the previous studies $[1-5,7,8,10-13]$. Based on the mean values of the objective function, all seven optimizer performances are greatly improved when implementing the path repairing algorithm as summarized in Table 3. All the optimizers can find the feasible solutions for all 30 runs. The optimizer that gives the best results is TLBO for both the mean value $\left(f_{\text {obj }}=0.3705\right)$ and the best result $\left(f_{\text {obj }}=0.0010\right)$. The second and third best optimizers are ABC and GWO, respectively. The worst performer in this case, according to Mean, is SCA. In Table 2, only ABC can search for feasible solutions for all 30 runs. Based on the mean objective values, the performance of all optimizers deteriorates compared to the results from using PRA in Table 1. The best in cases of using the exterior penalty function technique is $\mathrm{ABC}$, while the second best is GWO as it can search for feasible solutions for 29 from 30 runs with lower mean objective value compared to JADE. Figures 2 and 3 show the path traced by the coupler point of the best solution and the kinematic diagram of best linkage, respectively. It is found that TLBO with the novel design repairing technique gives the best result (Error $=$ $0.0122)$ better than previous work $[5,14]$ (Error $=0.1227$ (DE), and 0.0166 (DE), respectively). This design result cannot be compared with the previous work [15] due to the number of evaluation of the present work that is lower than ten times when compared with the reference. The best actual path as shown in Figure 3 is closer to the target path than in previous work $[5,14]$ and comparable with [15]. The four-bar linkage obtained from the best solution as shown in Figure 3 can completely rotate in an ascending direction.

For Case 2 with the number of target points at 6 , the results obtained from using the various MHs with PRA and the penalty function technique are shown in Tables 4 and 5, respectively. The coupler curves and the best linkages obtained from TLBO are shown in Figures 4 and 5, respectively. In this case, all MHs are significantly improved by using PRA based on the mean objective function values. They can find feasible solutions for all runs with the given predefined number of function evaluations. Note that PBIL using PRA has higher mean value compared to that using the penalty function technique, but this is computed from only 21 successful runs when using the penalty function technique. Based on using PRA in Table 4, the best method is TLBO while the second and third best algorithms are JADE and PBIL, respectively. The worst optimizer is SCA according to the mean value. In Table 5, based on using the penalty function technique, the top three performers are JADE, GWO, and $\mathrm{ABC}$ in that order. In this design case, TLBO produces the best solutions for both constraint 
TABle 1: Comparative results for Case 1 with a novel path repairing technique.

\begin{tabular}{|c|c|c|c|c|c|c|c|}
\hline \multicolumn{8}{|c|}{ Case 1: path generation without prescribed timing } \\
\hline Parameters & $\mathrm{ABC}$ & JADE & PBIL & TLBO & ACOR & GWO & SCA \\
\hline$r_{1}$ & 39.7861 & 52.4762 & 53.7995 & 57.9042 & 59.4760 & 33.4305 & 28.3704 \\
\hline$r_{2}$ & 10.1505 & 14.3110 & 9.4303 & 8.5359 & 9.1050 & 9.8739 & 5.8563 \\
\hline$r_{3}$ & 50.4447 & 48.0989 & 44.1070 & 20.5846 & 51.8860 & 38.5048 & 60.0000 \\
\hline$r_{4}$ & 35.2568 & 47.5363 & 46.5599 & 55.5223 & 20.4784 & 53.3193 & 38.7556 \\
\hline$r_{c x}$ & 45.1511 & 30.2155 & 47.8722 & 5.0306 & -6.9474 & 37.5632 & 60.0000 \\
\hline$r_{c y}$ & 7.2885 & -14.8707 & 32.8201 & 35.6648 & -21.9732 & -10.3487 & -60.0000 \\
\hline$x_{2}$ & 50.7782 & 11.7094 & 60.0000 & 47.0842 & -8.8106 & -5.2992 & -60.0000 \\
\hline$y_{2}$ & -0.9366 & -3.1269 & -8.8920 & 12.5554 & 28.8944 & 59.6847 & 60.0000 \\
\hline$\theta_{0}$ & 82.2709 & 51.1387 & 45.4292 & 355.3772 & 96.7425 & 234.8353 & 0.0000 \\
\hline$\theta_{2}^{1}$ & 288.6774 & 313.0937 & 352.6949 & 9.4470 & 276.1848 & 334.3267 & 0.0000 \\
\hline$\theta_{2}^{2}$ & 18.7094 & 338.5807 & 24.3156 & 23.8648 & 298.9037 & 5.9786 & 71.2728 \\
\hline$\theta_{2}^{3}$ & 39.4220 & 359.8582 & 29.9612 & 37.4934 & 337.6217 & 31.4398 & 86.9490 \\
\hline$\theta_{2}^{4}$ & 57.0069 & 18.5113 & 56.1299 & 51.6572 & 358.4935 & 61.3596 & 103.9052 \\
\hline$\theta_{2}^{5}$ & 75.0134 & 44.8517 & 73.4859 & 67.9991 & 33.9200 & 90.2248 & 130.0967 \\
\hline$\theta_{2}^{6}$ & 103.2820 & 85.9854 & 136.6204 & 91.0354 & 57.5750 & 139.0187 & 166.4231 \\
\hline Min & 0.6374 & 1.8255 & 6.7539 & 0.0010 & 49.1961 & 0.3271 & 42.5282 \\
\hline Mean & 4.1297 & 7.5933 & 20.0681 & 0.3705 & 73.1582 & 8.5501 & 144.5432 \\
\hline Max & 11.9015 & 17.0022 & 47.5797 & 2.7424 & 101.7039 & 94.4204 & 391.5969 \\
\hline Std & 2.7684 & 3.4092 & 10.1640 & 0.6667 & 16.1132 & 17.0012 & 94.5604 \\
\hline Error & 0.2380 & 0.4502 & 0.8232 & 0.0122 & 2.6807 & 0.2107 & 2.6387 \\
\hline Success & 30 & 30 & 30 & 30 & 30 & 30 & 30 \\
\hline
\end{tabular}

Success $=$ number of successful runs.

TABLe 2: Comparative results for Case 1 with a penalty technique.

\begin{tabular}{|c|c|c|c|c|c|c|c|}
\hline \multicolumn{8}{|c|}{ Case 1: path generation without prescribed timing } \\
\hline Parameters & $\mathrm{ABC}$ & JADE & PBIL & TLBO & ACOR & GWO & SCA \\
\hline$\overline{r_{1}}$ & 38.2010 & 31.9116 & 34.6475 & 59.9108 & 22.0645 & 43.6675 & 60.0000 \\
\hline$r_{2}$ & 8.6064 & 7.4478 & 6.4582 & 29.9172 & 5.0000 & 5.3499 & 5.0000 \\
\hline$r_{3}$ & 22.4589 & 43.6552 & 21.6196 & 59.9967 & 60.0000 & 26.5380 & 47.3444 \\
\hline$r_{4}$ & 33.2769 & 31.8355 & 23.7955 & 54.2963 & 60.0000 & 22.5663 & 60.0000 \\
\hline$r_{c x}$ & 20.0224 & 9.4994 & 30.1437 & 59.7723 & 4.2836 & -17.2375 & 60.0000 \\
\hline$r_{c y}$ & 24.8915 & 25.3461 & -25.6646 & 11.4222 & 11.0367 & 43.5804 & -60.0000 \\
\hline$x_{2}$ & -5.1270 & -0.4792 & 51.7915 & 41.5439 & 32.8024 & 59.9713 & -60.0000 \\
\hline$y_{2}$ & 52.6358 & 38.6191 & 54.5888 & -2.4092 & 29.3709 & 10.5553 & -0.6986 \\
\hline$\theta_{0}$ & 212.3407 & 224.9538 & 211.7426 & 47.8784 & 15.3431 & 16.7149 & 0.0000 \\
\hline$\theta_{2}^{1}$ & 307.2217 & 241.9357 & 211.4543 & 304.0545 & 159.2096 & 342.4006 & 21.6624 \\
\hline$\theta_{2}^{2}$ & 357.3637 & 226.6521 & 243.5720 & 347.0862 & 246.5489 & 15.3455 & 201.7648 \\
\hline$\theta_{2}^{3}$ & 26.8761 & 204.4762 & 287.1713 & 359.9988 & 253.5892 & 37.5406 & 75.3665 \\
\hline$\theta_{2}^{4}$ & 52.9043 & 174.1217 & 311.7283 & 9.0531 & 299.5365 & 60.6278 & 3.1728 \\
\hline$\theta_{2}^{5}$ & 80.7316 & 141.3107 & 333.2289 & 16.0694 & 0.0000 & 91.7984 & 0.0000 \\
\hline$\theta_{2}^{6}$ & 110.8412 & 131.9133 & 58.1996 & 22.0740 & 111.4815 & 134.0130 & 360.0000 \\
\hline Min & 0.9797 & 23.1751 & 23.1950 & 0.2399 & 523.5085 & 0.8853 & 358.3843 \\
\hline Mean & 23.4148 & 297.8795 & 133.3815 & 175.9177 & 1005.078 & 147.9292 & 452.8526 \\
\hline Max & 78.2443 & 1151.615 & 247.9302 & 1106.170 & 1541.802 & 399.6278 & 562.5967 \\
\hline Std & 18.2255 & 242.1490 & 74.2606 & 226.5618 & 236.8720 & 120.4374 & 102.9596 \\
\hline Error & 0.3545 & 1.6529 & 1.7811 & 0.1808 & 7.9631 & 0.3532 & 5.9932 \\
\hline Success & 30 & 29 & 6 & 26 & 22 & 29 & 3 \\
\hline
\end{tabular}

Success $=$ number of successful runs.

handling techniques. For this case, it is proved that using the second repairing technique (Algorithm 2) is better than the penalty function technique. The best optimizer is TLBO as with the first case which gives the best error result as 0.3073 , while the results from previous work $[5,13,14]$ are error $=$
2.3496 (DE), 2.5998 (ICA), and 1.6063 (JADE), respectively. The best actual path as shown in Figure 4 is closer with the target path than the previous work $[5,13,14]$. The fourbar linkage obtained from the best solution as shown in Figure 5 can completely rotate in an ascending direction in 
Table 3: Comparative Min and Mean for Cases 1-3 with a novel path repairing technique and a penalty technique.

\begin{tabular}{|c|c|c|c|c|c|c|c|c|c|}
\hline Case number & With & Parameters & $\mathrm{ABC}$ & JADE & PBIL & TLBO & ACOR & GWO & SCA \\
\hline \multirow{6}{*}{1} & \multirow{6}{*}{ Penalty technique } & Min & 0.6374 & 1.8255 & 6.7539 & 0.0010 & 49.1961 & 0.3271 & 42.5282 \\
\hline & & Mean & 4.1297 & 7.5933 & 20.0681 & 0.3705 & 73.1582 & 8.5501 & 144.5432 \\
\hline & & Success & 30 & 30 & 30 & 30 & 30 & 30 & 30 \\
\hline & & Min & 0.9797 & 23.1751 & 23.1950 & 0.2399 & 523.5085 & 0.8853 & 358.3843 \\
\hline & & Mean & 23.4148 & 297.8795 & 133.3815 & 175.9177 & 1005.078 & 147.9292 & 452.8526 \\
\hline & & Success & 30 & 29 & 6 & 26 & 22 & 29 & 3 \\
\hline \multirow{6}{*}{2} & \multirow{3}{*}{ PRA } & Min & 7.3696 & 0.7614 & 2.7790 & 0.7614 & 3.6197 & 1.0934 & 22.7680 \\
\hline & & Mean & 31.4436 & 13.3175 & 24.3873 & 9.2918 & 31.1379 & 27.5451 & 147.5295 \\
\hline & & Success & 30 & 30 & 30 & 30 & 30 & 30 & 30 \\
\hline & \multirow{3}{*}{ Penalty technique } & Min & 6.1512 & 8.5978 & 2.2458 & 0.7614 & 24.4416 & 2.6452 & 43.5787 \\
\hline & & Mean & 58.0879 & 35.9131 & 20.6274 & 45.8361 & 98.6229 & 39.3821 & 340.6984 \\
\hline & & Success & 30 & 30 & 21 & 29 & 30 & 30 & 30 \\
\hline \multirow{6}{*}{3} & \multirow{6}{*}{ Penalty technique } & Min & 1.1331 & 2.2551 & 41.9444 & 0.0192 & 156.6075 & 26.8977 & 306.5633 \\
\hline & & Mean & 7.7983 & 9.2387 & 116.5084 & 2.5706 & 310.5264 & 70.7824 & 568.2001 \\
\hline & & Success & 30 & 30 & 30 & 30 & 30 & 30 & 30 \\
\hline & & Min & 1.0714 & 1212.697 & 42.6040 & 352.3451 & 0.0000 & 251.2720 & 0.0000 \\
\hline & & Mean & 38.2367 & 1212.697 & 402.5957 & 576.6454 & 0.0000 & 493.2413 & 0.0000 \\
\hline & & Success & 29 & 1 & 4 & 10 & 0 & 15 & 0 \\
\hline
\end{tabular}

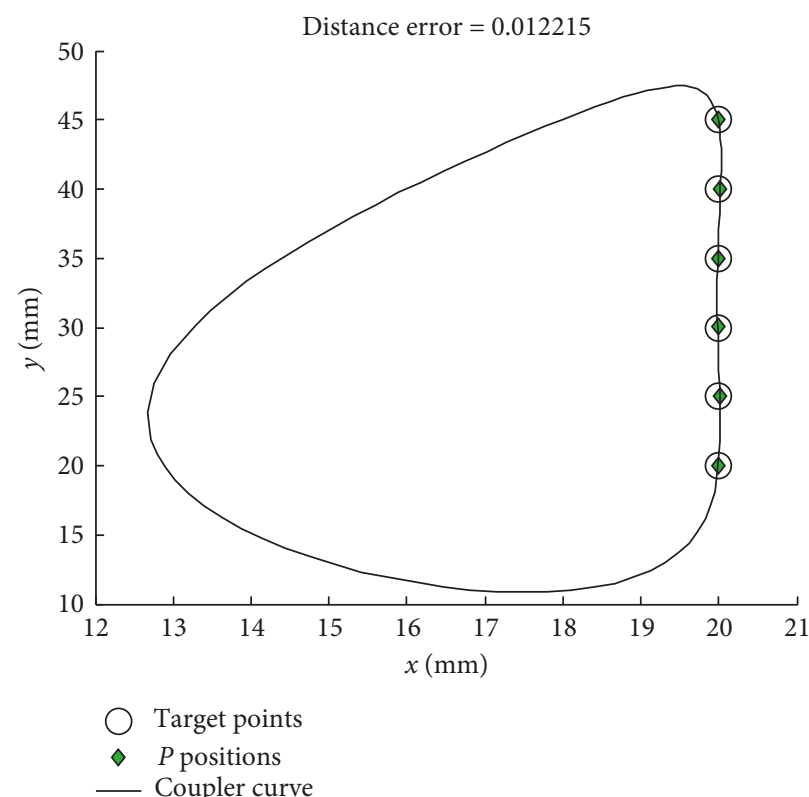

Figure 2: Best coupler curve obtained in Case 1.

accordance with the prescribed timing and the circular target path.

In Case 3 with the prescribed curve with 10 points, the results are obtained by those algorithms with PRA, and the penalty function techniques are shown in Tables 6 and 7 , respectively. The coupler curve and the best linkage are shown in Figures 6 and 7, respectively. From the results, it is found that TLBO gives the best results for both the mean objective function value and the best result when using PRA. Similarly to the previous two cases, all MHs are greatly improved when using PRA. All the methods can find feasible solutions for all runs with using PRA. The top three performers in cases of using PRA are TLBO, ABC, and JADE in that order while the worst method is SCA based on the mean objective values. In the results of using penalty function technique, ACOR, and SCA cannot find a feasible solution. The best method when using penalty function technique is $\mathrm{ABC}$ while the performance of others cannot be evaluated as they can search for feasible solutions for a few optimization runs. The best result from TLBO gives an average distance error of 0.0324 while the previous work $[5,14]$ had error $=1.9523(\mathrm{DE})$, and error $=0.1641$ (DE), respectively. It means that the best actual path as shown in Figure 6 is closer with the target path than the previous work [5, 14]. The linkage obtained from the best solution as shown in Figure 7 can completely rotate in an ascending direction in accordance with the elliptic target path. 




Figure 3: Best mechanism obtained in Case 1.

TABLE 4: Comparative results of Case 2 with a novel path repairing technique.

\begin{tabular}{|c|c|c|c|c|c|c|c|}
\hline \multicolumn{8}{|c|}{ Case 2: path generation with prescribed timing } \\
\hline Parameters & $\mathrm{ABC}$ & JADE & PBIL & TLBO & ACOR & GWO & SCA \\
\hline$r_{1}$ & 50.0000 & 47.3318 & 37.3034 & 47.3319 & 47.0787 & 48.7592 & 23.7320 \\
\hline$r_{2}$ & 8.7953 & 8.9594 & 9.7011 & 8.9594 & 9.5109 & 8.8102 & 6.4302 \\
\hline$r_{3}$ & 17.5326 & 26.1415 & 27.5790 & 26.1415 & 23.8293 & 23.8043 & 43.5310 \\
\hline$r_{4}$ & 50.0000 & 50.0000 & 40.1382 & 50.0000 & 44.9649 & 45.6184 & 37.4296 \\
\hline$r_{c x}$ & 35.9767 & 43.5295 & 38.4166 & 43.5296 & 47.1950 & 49.8852 & 15.6308 \\
\hline$r_{c y}$ & -7.7228 & -27.9916 & -31.4443 & -27.9914 & -14.3055 & -13.8744 & -50.0000 \\
\hline$x_{2}$ & 16.0681 & 16.8224 & 16.2277 & 16.8224 & 17.8426 & 16.9234 & 14.9035 \\
\hline$y_{2}^{2}$ & -34.3113 & -50.0000 & -48.1851 & -50.0000 & -47.7051 & -49.9768 & -50.0000 \\
\hline$\theta_{0}$ & 31.3196 & 48.3625 & 59.5519 & 48.3624 & 45.2184 & 44.2373 & 107.4695 \\
\hline Min & 7.3696 & 0.7614 & 2.7790 & 0.7614 & 3.6197 & 1.0934 & 22.7680 \\
\hline Mean & 31.4436 & 13.3175 & 24.3873 & 9.2918 & 31.1379 & 27.5451 & 147.5295 \\
\hline Max & 52.3994 & 26.1423 & 92.0479 & 17.8459 & 107.1751 & 192.4375 & 660.2919 \\
\hline Std & 13.9207 & 7.9546 & 18.4786 & 8.1308 & 23.2712 & 36.4676 & 141.0674 \\
\hline Error & 1.0356 & 0.3073 & 0.6318 & 0.3073 & 0.6749 & 0.3723 & 1.6759 \\
\hline Success & 30 & 30 & 30 & 30 & 30 & 30 & 30 \\
\hline
\end{tabular}

Success $=$ number of successful runs.

TABLE 5: Comparative result of Case 2 with a penalty technique.

\begin{tabular}{|c|c|c|c|c|c|c|c|}
\hline \multicolumn{8}{|c|}{ Case 2: path generation with prescribed timing } \\
\hline Parameters & $\mathrm{ABC}$ & $\mathrm{JADE}$ & PBIL & TLBO & ACOR & GWO & SCA \\
\hline$r_{1}$ & 40.7603 & 32.5637 & 50.0000 & 47.3284 & 47.6986 & 49.0512 & 25.7137 \\
\hline$r_{2}$ & 5.8249 & 7.2738 & 8.9238 & 8.9594 & 6.4661 & 7.9356 & 5.0000 \\
\hline$r_{3}$ & 9.9768 & 22.0128 & 23.9506 & 26.1434 & 21.0563 & 21.2936 & 25.7783 \\
\hline$r_{4}$ & 38.6534 & 31.9534 & 46.8513 & 50.0000 & 48.2501 & 41.6373 & 38.5477 \\
\hline$r_{c x}$ & 28.0556 & 42.1632 & 48.6935 & 43.5248 & 46.0280 & 50.0000 & 15.3979 \\
\hline$r_{c y}$ & -5.7200 & -28.2724 & -16.8126 & -27.9988 & -23.7354 & 3.4330 & -50.0000 \\
\hline$x_{2}$ & 12.9887 & 12.8441 & 17.1186 & 16.8220 & 12.5560 & 16.2388 & 12.3447 \\
\hline$y_{2}$ & -24.4168 & -48.8226 & -49.3931 & -50.0000 & -50.0000 & -47.9755 & -50.0000 \\
\hline$\theta_{0}$ & 34.5068 & 57.2910 & 46.9004 & 48.3661 & 41.6402 & 36.6935 & 67.0180 \\
\hline Min & 6.1512 & 8.5978 & 2.2458 & 0.7614 & 24.4416 & 2.6452 & 43.5787 \\
\hline Mean & 58.0879 & 35.9131 & 20.6274 & 45.8361 & 98.6229 & 39.3821 & 340.6984 \\
\hline Max & 168.5279 & 97.6451 & 86.4201 & 609.1712 & 173.0658 & 108.0179 & 1064.047 \\
\hline Std & 44.3671 & 17.0311 & 20.9987 & 139.0780 & 39.9827 & 32.7100 & 265.7480 \\
\hline Error & 0.8997 & 1.0665 & 0.5467 & 0.3073 & 1.9211 & 0.6451 & 2.5063 \\
\hline Success & 30 & 30 & 21 & 29 & 30 & 30 & 30 \\
\hline
\end{tabular}

Success $=$ number of successful runs. 




Figure 4: Best coupler curve obtained in Case 2.

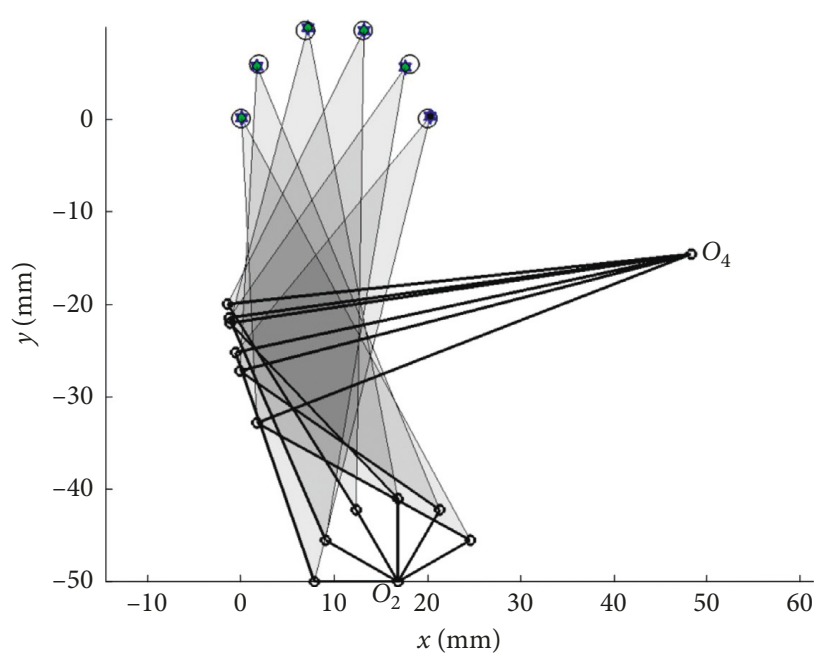

Figure 5: Best mechanism obtained in Case 2.

Table 3 gives a summary of the comparative performance of the various metaheuristics for solving the fourbar linkage path synthesis using the new constraint handling technique and the classical exterior penalty function technique. It shows that the results from using PRA are totally superior to those obtained from using the penalty function.

It is shown that TLBO with PRA is superior to the other MHs using the penalty technique. In this study, the Friedman test and the Tukey-Kramer test are used for comparing the results. Based on the Friedman test, TLBO ranks 1st whereas the second best is JADE at $p$ value $(0.02)<\alpha(0.05)$ as shown in Table 8. It can be summarized that TLBO is the best performer for solving the four-bar path synthesis problems cases 1-3. Based on the Tukey-Kramer test, the mean column ranks of TLBO are significantly different from SCA.
The mean values obtained from using all optimizers of the test problems with PRA and the penalty function technique are shown in Table 3. In the statistical study, the results from ACOR and SCA are discarded because they cannot find any feasible solution in Case 3 (without using PRA). This table shows that MHs using the PRA approach give better mean than their counterparts that employ the penalty function technique for all test problems. The of average results of Friedman test are given in Table 9 which shows that MHs with the PRA technique significantly outperforms those using the penalty function technique at $p$ value $(0.00016)<\alpha(0.05)$.

Figures 8-10 illustrate the search histories of the best runs of TLBO for Case 1, Case 2, and Case 3, respectively. The search history compares the best runs obtained from using the penalty function technique and the path repairing 
TABLE 6: Comparative results of Case 3 with a novel path repairing technique.

\begin{tabular}{|c|c|c|c|c|c|c|c|}
\hline \multicolumn{8}{|c|}{ Case 3: path generation without prescribed timing } \\
\hline Parameters & $\mathrm{ABC}$ & JADE & PBIL & TLBO & ACOR & GWO & SCA \\
\hline$r_{1}$ & 80.0000 & 79.9162 & 69.0571 & 42.0053 & 66.5453 & 43.5326 & 80.0000 \\
\hline$r_{2}$ & 8.1263 & 9.7027 & 10.0219 & 8.0876 & 10.6498 & 19.6630 & 5.1563 \\
\hline$r_{3}$ & 80.0000 & 79.6300 & 80.0000 & 28.2660 & 65.3047 & 66.2180 & 64.8837 \\
\hline$r_{4}$ & 51.8439 & 22.1050 & 66.3977 & 24.1099 & 62.9137 & 42.3820 & 61.7959 \\
\hline$r_{c x}$ & -19.3276 & 13.4224 & -6.0994 & -4.4860 & 6.0790 & 25.1410 & -40.4117 \\
\hline$r_{c y}$ & -11.0023 & -10.1904 & -27.8102 & -4.7935 & -6.9436 & -25.6124 & -80.0000 \\
\hline$x_{2}$ & -1.2988 & 25.0942 & -13.1694 & 11.1765 & 2.5885 & -16.7352 & 25.4964 \\
\hline$y_{2}$ & 29.1004 & 3.1796 & 26.4926 & 3.5870 & 7.9850 & 8.3961 & -80.0000 \\
\hline$\theta_{0}$ & 53.9222 & 176.9942 & 12.1922 & 203.0714 & 0.0000 & 10.8144 & 162.9594 \\
\hline$\theta_{2}^{1}$ & 349.6393 & 0.0377 & 8.8375 & 359.1656 & 52.8186 & 75.4675 & 47.9605 \\
\hline$\theta_{2}^{2}$ & 35.6886 & 31.3880 & 45.3792 & 38.0948 & 76.7817 & 88.6364 & 77.3485 \\
\hline$\theta_{2}^{3}$ & 77.1187 & 73.5207 & 79.6200 & 76.7126 & 111.7879 & 107.0112 & 78.7932 \\
\hline$\theta_{2}^{4}$ & 115.8808 & 117.4221 & 91.7790 & 115.5382 & 139.0174 & 129.7889 & 102.9839 \\
\hline$\theta_{2}^{5}$ & 153.8239 & 160.1615 & 140.7557 & 154.7689 & 168.2661 & 155.2651 & 132.9758 \\
\hline$\theta_{2}^{6}$ & 193.8898 & 201.3488 & 196.8064 & 196.1914 & 196.3339 & 189.1377 & 158.4142 \\
\hline$\theta_{2}^{7}$ & 234.8222 & 238.3213 & 225.1631 & 236.9290 & 236.0848 & 228.5280 & 178.2059 \\
\hline$\theta_{2}^{8}$ & 275.8371 & 275.8487 & 272.5570 & 277.6224 & 269.5633 & 255.6323 & 216.7998 \\
\hline$\theta_{2}^{9}$ & 311.5613 & 312.9753 & 298.1521 & 319.1629 & 298.2293 & 276.9471 & 227.5118 \\
\hline$\theta_{2}^{10}$ & 348.9137 & 355.2115 & 360.0000 & 359.1397 & 322.3910 & 290.1490 & 239.6187 \\
\hline Min & 1.1331 & 2.2551 & 41.9444 & 0.0192 & 156.6075 & 26.8977 & 306.5633 \\
\hline Mean & 7.7983 & 9.2387 & 116.5084 & 2.5706 & 310.5264 & 70.7824 & 568.2001 \\
\hline Max & 30.3123 & 25.7054 & 306.4299 & 28.5182 & 551.2737 & 234.2163 & 1144.216 \\
\hline Std & 7.1036 & 4.9496 & 62.3238 & 6.4004 & 83.5219 & 43.5185 & 153.6016 \\
\hline Error & 0.2925 & 0.4315 & 1.8480 & 0.0324 & 3.5846 & 1.4398 & 5.2041 \\
\hline Success & 30 & 30 & 30 & 30 & 30 & 30 & 30 \\
\hline
\end{tabular}

Success $=$ number of successful runs.

TABLE 7: Comparative results of Case 3 with a penalty technique.

\begin{tabular}{|c|c|c|c|c|c|c|c|}
\hline \multicolumn{8}{|c|}{ Case 3: path generation without prescribed timing } \\
\hline Parameters & $\mathrm{ABC}$ & JADE & PBIL & TLBO & ACOR & GWO & SCA \\
\hline$r_{1}$ & 68.1732 & 19.9901 & 40.6029 & 17.7554 & 0.0000 & 59.7863 & 0.0000 \\
\hline$r_{2}$ & 9.4618 & 5.8357 & 8.1608 & 5.0231 & 0.0000 & 5.0000 & 0.0000 \\
\hline$r_{3}$ & 80.0000 & 43.1677 & 50.0664 & 32.9105 & 0.0000 & 74.9475 & 0.0000 \\
\hline$r_{4}$ & 80.0000 & 50.3385 & 34.8760 & 31.2567 & 0.0000 & 30.6040 & 0.0000 \\
\hline$r_{c x}$ & -1.1813 & 18.7411 & -0.3738 & 5.8641 & 0.0000 & 15.4730 & 0.0000 \\
\hline$r_{c y}$ & -13.1623 & -25.0130 & 26.8058 & 24.6039 & 0.0000 & 80.0000 & 0.0000 \\
\hline$x_{2}$ & -1.1627 & 41.0708 & 26.7918 & 10.3944 & 0.0000 & 30.7488 & 0.0000 \\
\hline$y_{2}$ & 16.3687 & 12.8362 & 30.3681 & -16.0968 & 0.0000 & -70.6740 & 0.0000 \\
\hline$\theta_{0}$ & 360.0000 & 134.8235 & 99.1181 & 309.4384 & 0.0000 & 4.4956 & 0.0000 \\
\hline$\theta_{2}^{1}$ & 0.0000 & 172.6179 & 32.5643 & 351.2755 & 0.0000 & 360.0000 & 0.0000 \\
\hline$\theta_{2}^{2}$ & 37.4238 & 325.2499 & 72.4952 & 52.7076 & 0.0000 & 34.6517 & 0.0000 \\
\hline$\theta_{2}^{3}$ & 75.2680 & 337.0868 & 88.5868 & 94.6252 & 0.0000 & 102.8443 & 0.0000 \\
\hline$\theta_{2}^{4}$ & 114.8952 & 20.4030 & 112.0993 & 143.0734 & 0.0000 & 135.9833 & 0.0000 \\
\hline$\theta_{2}^{5}$ & 152.8726 & 182.4764 & 173.3556 & 190.6071 & 0.0000 & 164.5501 & 0.0000 \\
\hline$\theta_{2}^{6}$ & 191.5270 & 331.0880 & 201.7909 & 317.9020 & 0.0000 & 186.7582 & 0.0000 \\
\hline$\theta_{2}^{7}$ & 232.1421 & 82.2786 & 258.3224 & 129.4480 & 0.0000 & 188.6709 & 0.0000 \\
\hline$\theta_{2}^{8}$ & 274.8734 & 134.4962 & 280.6872 & 300.9699 & 0.0000 & 8.0308 & 0.0000 \\
\hline$\theta_{2}^{9}$ & 314.3132 & 241.5786 & 318.6172 & 335.0357 & 0.0000 & 19.9826 & 0.0000 \\
\hline$\theta_{2}^{10}$ & 0.0000 & 303.1435 & 43.3601 & 357.2376 & 0.0000 & 51.8922 & 0.0000 \\
\hline Min & 1.0714 & 1212.697 & 42.6040 & 352.3451 & 0.0000 & 251.2720 & 0.0000 \\
\hline Mean & 38.2367 & 1212.697 & 402.5957 & 576.6454 & 0.0000 & 493.2413 & 0.0000 \\
\hline Max & 110.6796 & 1212.697 & 781.4347 & 990.3743 & 0.0000 & 1901.836 & 0.0000 \\
\hline Std & 25.2906 & 0.0000 & 398.2755 & 210.1756 & 0.0000 & 402.5266 & 0.0000 \\
\hline Error & 0.3074 & 10.1061 & 1.9909 & 4.9788 & 0.0000 & 4.6902 & 0.0000 \\
\hline Success & 29 & 1 & 4 & 10 & 0 & 15 & 0 \\
\hline
\end{tabular}

Success $=$ number of successful runs. 


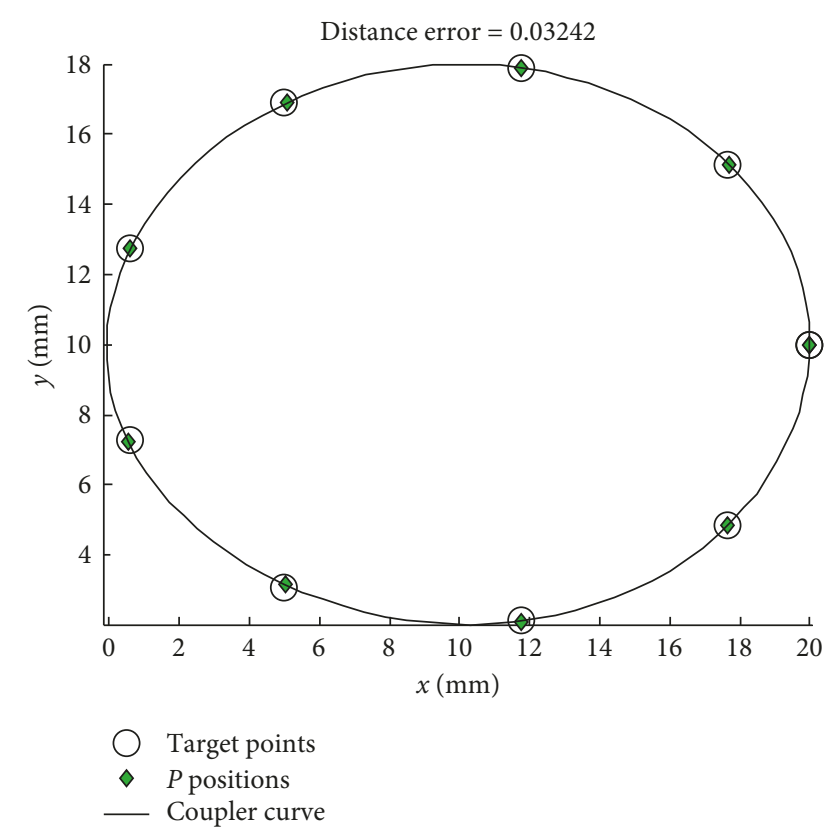

Figure 6: Best coupler curve obtained in Case 3.



Figure 7: Best mechanism obtained in Case 3.

TABle 8: Average ranking and $p$ value of performance index of MHs achieved by Friedman test.

\begin{tabular}{lccccccc}
\hline \multicolumn{7}{c}{ Average ranking of each algorithm Friedman } & $p$ \\
ABC & JADE & PBIL & TLBO & ACOR & GWO & SCA & value \\
\hline 3.3333 & $\begin{array}{c}2.6667 \\
(3)\end{array}$ & $\begin{array}{c}4.3333 \\
(5)\end{array}$ & 1 (1) & $\begin{array}{c}5.6667 \\
(6)\end{array}$ & $4(4)$ & $7(7)$ & 0.02 \\
\hline
\end{tabular}

TABle 9: Performance comparison of each case study with and without a novel path repairing technique for all algorithms.

\begin{tabular}{llc}
\hline$p$ value & $\begin{array}{c}\text { Average ranking of each technique Friedman } \\
\text { With PRA }\end{array}$ & Without PRA \\
\hline 0.00016 & $1.0556(1)$ & $1.9444(2)$ \\
\hline
\end{tabular}

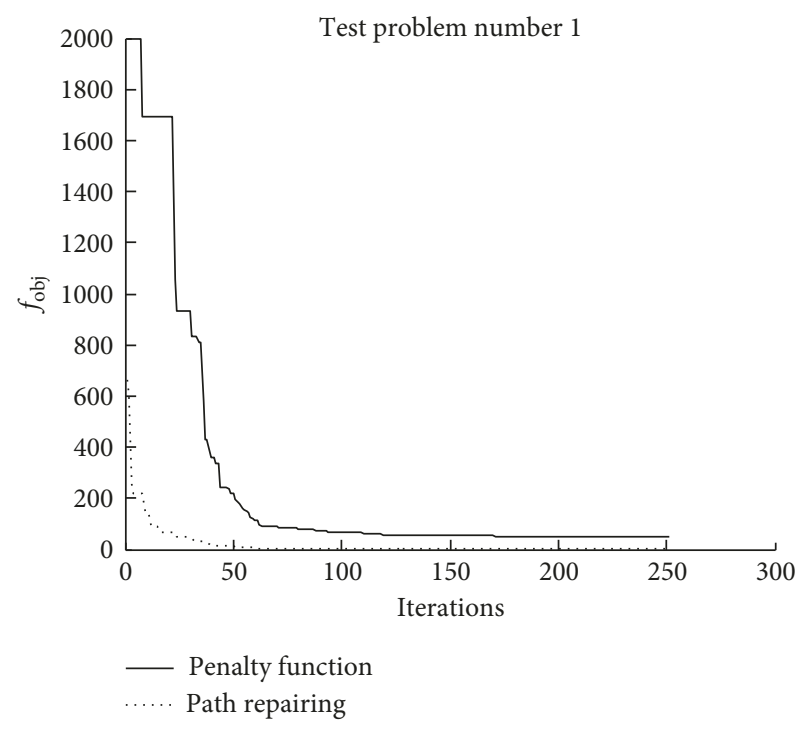

Figure 8: Search history of the best result obtained in Case 1.

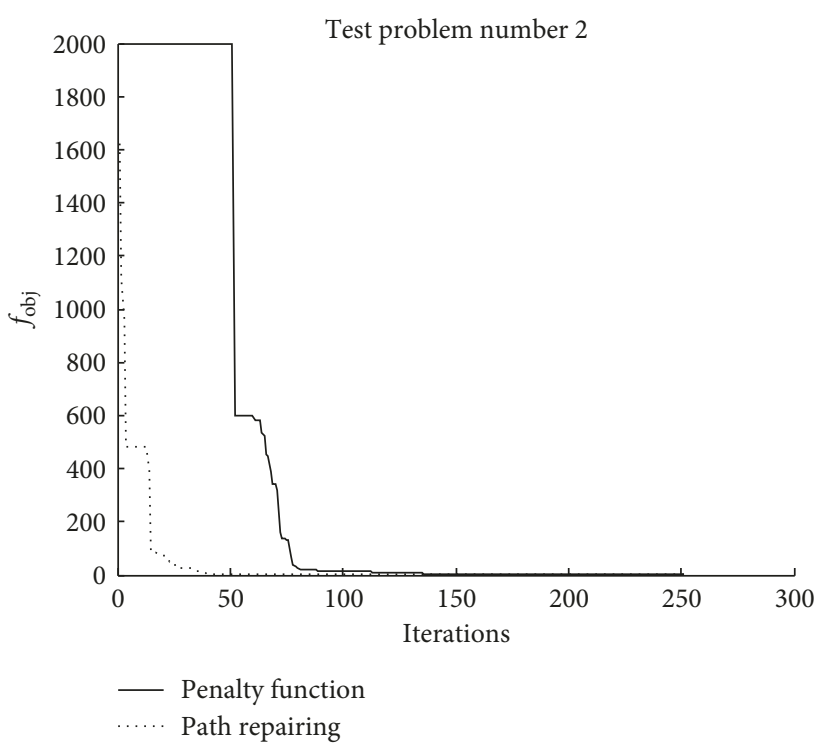

Figure 9: Search history of the best result obtained in Case 2.

algorithm. It can be seen that even though TLBO using PRA started with the worse solution (Case 3), the path repairing technique still guided the optimizer to converge considerably faster than when using the penalty function technique. All three figures show the superiority of the proposed path repairing technique for four-bar linkage path synthesis.

\section{Discussion}

Further discussion is provided in order to investigate the behavior of the proposed constraint handling technique PRA and why it is efficient when used with TLBO. Figure 11 shows the search history of the best runs of TLBO for the case 1 problem using PRA and the penalty function (PF) technique. The figure displays the number of iterations versus the number of infeasible solutions for both runs. The 


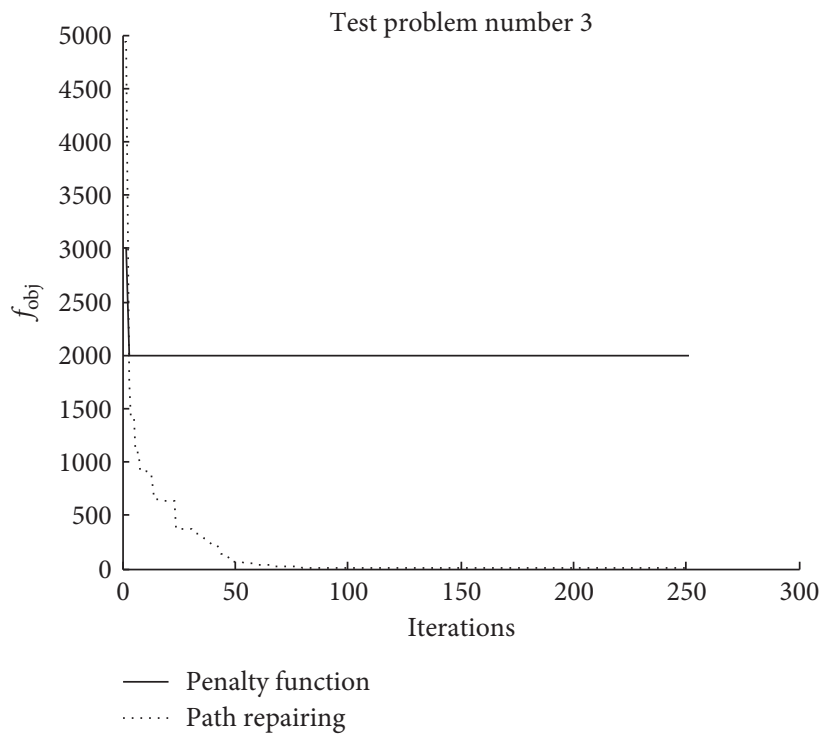

Figure 10: Search history of the best result obtained in Case 3.

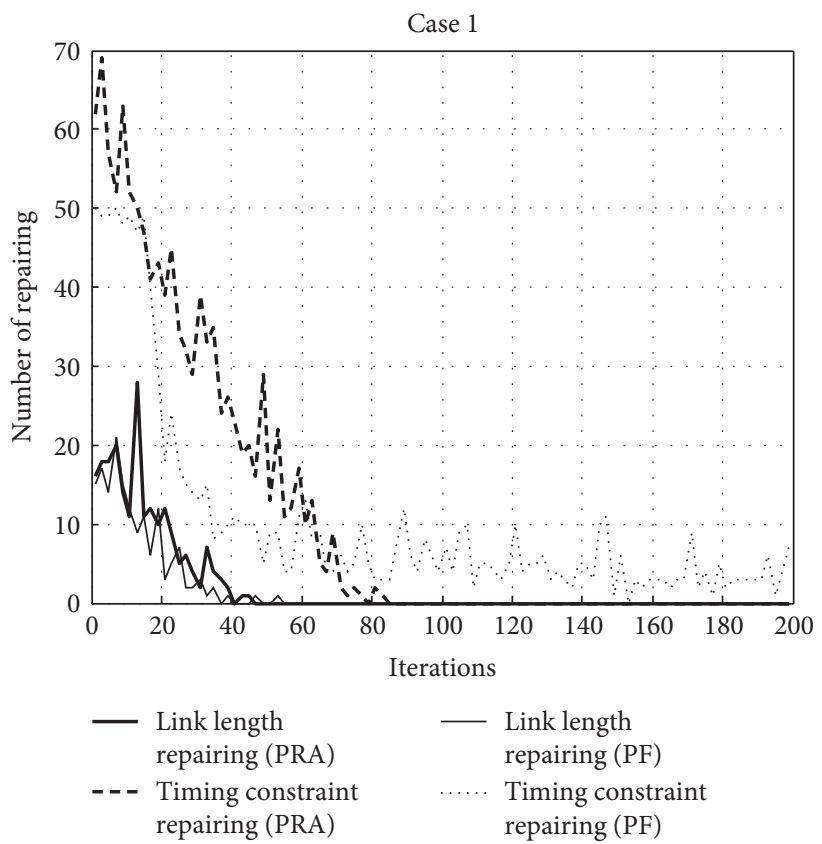

Figure 11: Repairing and penalty function histories of Case 1 for 200 iterations.

illustration also separates the number of infeasible solutions due to the timing constraint and those caused by the link length constraints. It can be seen that, with the use of PRA, infeasible solutions due to the link length constraints vanished after approximately 50 iterations. The same conclusion is applied in cases of using PF. By using PRA, the numbers of infeasible solutions due to the timing constraint disappear after around 85 iterations while the number of infeasible solutions due to the timing constraint when using PF cannot be suppressed throughout the search process. From this particular comparison, it is shown that the proposed PRA is efficient for dealing with both link length and timing constraints while the penalty function technique failed to solve the timing constraint problem.

Figure 12 shows the search history (objective function versus iterations) of the best runs of the three design cases from using TLBO in combination with PRA. In this figure, the best objective function values obtained from the teaching and learning phases of TLBO are plotted separately. It can be seen from all three path synthesis problems that the best results produced by the learning phase are equal to or better than those obtained from the teaching phase. This implies that the reproduction using the learning operator of TLBO works well with the proposed PRA. In TLBO, the teaching 


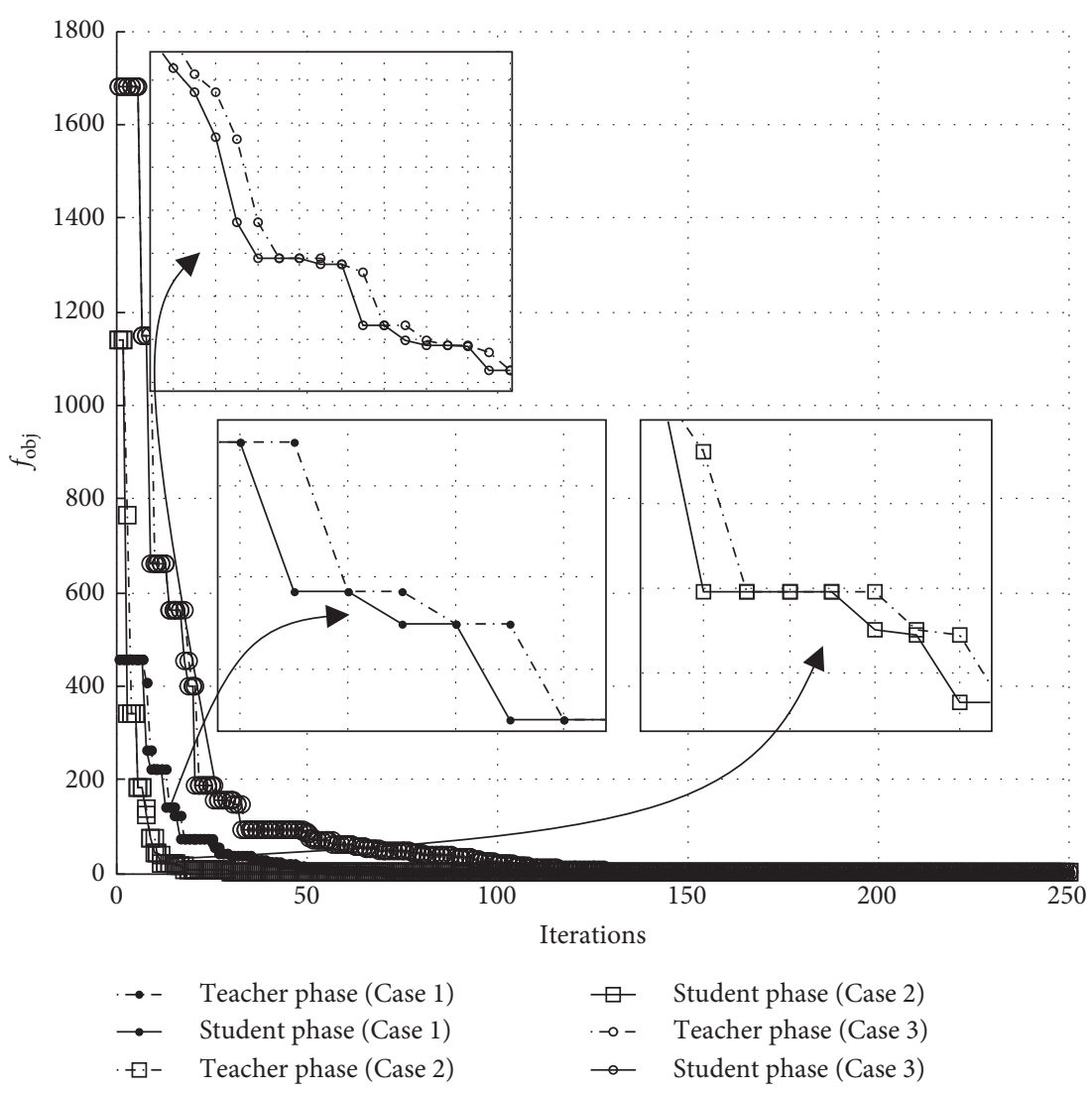

FIGURE 12: Teacher phase and student phase function evaluation history of Cases 1-3 for 500 iterations.

phase is used for exploitation while the learning phase emphasizes more on exploration. That means the proposed technique tends to be efficient with an exploration-based reproduction operator. As a result, further development of TLBO for path synthesis of a four-bar linkage can adapt from the original version by adding a self-adaptive strategy for population sizing.

\section{Conclusions}

This paper presents a technique to find the optimum parameters of a four-bar linkage for path synthesis using metaheuristics including ABC, JADE, PBIL, TLBO, ACOR, GWO, and SCA. The new technique, called a path repairing technique, is proposed to handle the synthesis constraints effectively. The comparative results of the three case studies show that the new path repairing technique is superior to the penalty function technique traditionally used in four-bar linkage path syntheses in the previous studies. The comparative performance of the metaheuristics shows that the TLBO with PRA is the most efficient method based on both convergence rate and consistency. The results in this work can consider as the baseline for developing an efficient optimizer for four-bar linkage path syntheses in the future. Any optimizer should be tested by running it many times to solve the synthesis problems, and the mean value of an objective function should be used as a performance indicator.

\section{Data Availability}

The data used to support the findings of this study are included within the article.

\section{Conflicts of Interest}

The authors declare that there are no conflicts of interest regarding the publication of this paper.

\section{Acknowledgments}

The authors are grateful for the financial support provided by the King Mongkut's Institute of Technology Ladkrabang, the Thailand Research Fund (RTA6180010), and the Postdoctoral Program from Research Affairs, Graduate School, Khon Kaen University (58225).

\section{References}

[1] A. Smaili and N. Diab, "Optimum synthesis of hybrid-task mechanisms using ant-gradient search method," Mechanism and Machine Theory, vol. 42, no. 1, pp. 115-130, 2007.

[2] J. A. Cabrera, F. Nadal, J. P. Muñoz, and A. Simon, "Multiobjective constrained optimal synthesis of planar mechanisms using a new evolutionary algorithm," Mechanism and Machine Theory, vol. 42, no. 7, pp. 791-806, 2007.

[3] R. R. Bulatović and S. R. Dordević, "On the optimum synthesis of a four-bar linkage using differential evolution and 
method of variable controlled deviations," Mechanism and Machine Theory, vol. 44, no. 1, pp. 235-246, 2009.

[4] N. Nariman-Zadeh, M. Felezi, A. Jamali, and M. Ganji, "Pareto optimal synthesis of four-bar mechanisms for path generation," Mechanism and Machine Theory, vol. 44, no. 1, pp. 180-191, 2009.

[5] S. K. Acharyya and M. Mandal, "Performance of EAs for fourbar linkage synthesis," Mechanism and Machine Theory, vol. 44, no. 9, pp. 1784-1794, 2009.

[6] A. F. Al-Dwairi, F. T. Dweiri, and O. M. Ashour, "A novicecentered decision-support system for type synthesis of function-generation mechanisms," Mechanism and Machine Theory, vol. 45, no. 9, pp. 1252-1268, 2010.

[7] W. Y. Lin, "A GA-DE hybrid evolutionary algorithm for path synthesis of four-bar linkage," Mechanism and Machine Theory, vol. 45, no. 8, pp. 1096-1107, 2010.

[8] J. A. Cabrera, A. Ortiz, F. Nadal, and J. J. Castillo, "An evolutionary algorithm for path synthesis of mechanisms," Mechanism and Machine Theory, vol. 46, no. 2, pp. 127-141, 2011.

[9] F. Peñuñuri, R. Peón-Escalante, C. Villanueva, and D. PechOy, "Synthesis of mechanisms for single and hybrid tasks using differential evolution," Mechanism and Machine Theory, vol. 46, no. 10, pp. 1335-1349, 2011.

[10] M. Khorshidi, M. Soheilypour, M. Peyro, A. Atai, and M. Shariat Panahi, "Optimal design of four-bar mechanisms using a hybrid multi-objective GA with adaptive local search," Mechanism and Machine Theory, vol. 46, no. 10, pp. 1453-1465, 2011.

[11] S. B. Mateka and G. R. Gogate, "Optimum synthesis of path generating four-bar mechanisms using differential evolution and a modified error function," Mechanism and Machine Theory, vol. 52, pp. 158-179, 2012.

[12] J. C. Heo and G. H. Yoon, "Size and configuration syntheses of rigid-link mechanisms with multiple rotary actuators using the constraint force design method," Mechanism and Machine Theory, vol. 64, pp. 18-38, 2013.

[13] S. Ebrahimi and P. Payvandy, "Efficient constrained synthesis of path generating four-bar mechanisms based on the heuristic optimization algorithms," Mechanism and Machine Theory, vol. 85, pp. 189-204, 2015.

[14] W. Phukaokaew, S. Sleesongsom, and S. Bureerat, "Four-bar linkage path synthesis using meta-heuristics-algorithm comparison," in Proceedings of the 7th TSME-ICOME, Chiang Mai, Thailand, December 2016.

[15] B. Hernández-Ocaña, M. P. D. Pozos-Parra, E. MezuraMontes, E. A. Portilla-Flores, E. Vega-Alvarado, and M. B. Calva-Yáñez, "Two-swim operators in the modified bacterial foraging algorithm for the optimal synthesis of fourbar mechanisms," Computational Intelligence and Neuroscience, vol. 2016, Article ID 4525294, 18 pages, 2016.

[16] S. Sleesongsom and S. Bureerat, "Optimal synthesis of fourbar linkage path generation through evolutionary computation," Research and Applications in Mechanical Engineering, vol. 3, no. 2, pp. 46-53, 2015.

[17] S. Sleesongsomand and S. Bureerat, "Four-bar linkage path generation through self-adaptive population size teachinglearning based optimization," Knowledge-Based Systems, vol. 135, pp. 180-191, 2017.

[18] S. Sleesongsom and S. Bureerat, "Multiobjective optimization of a steering linkage," Journal of Mechanical Science and Technology, vol. 30, no. 8, pp. 3681-3691, 2016.

[19] S. Bureerat and S. Srisomporn, "Optimum plate-fin heat sinks by using a multi-objective evolutionary algorithm," Engineering Optimization, vol. 42, no. 4, pp. 305-323, 2010.
[20] D. Karaboga and B. Akay, "A comparative study of Artificial Bee Colony algorithm," Applied Mathematics and Computation, vol. 214, no. 1, pp. 108-132, 2009.

[21] M. Dorigo and G. Di Caro, "The ant colony optimization metaheuristic," in New Ideas in Optimization, D. Corne, M. Dorigo, and F. Glover, Eds., pp. 11-32, McGraw Hill, London, UK, 1999.

[22] R. V. Rao and V. Patel, "An improved teaching-learningbased optimization algorithm for solving unconstrained optimization problems," Scientia Iranica, vol. 20, no. 3, pp. 710-720, 2012.

[23] J. Zhang and A. C. Sanderson, "JADE: adaptive differential evolution with optional external archive," IEEE Transaction on Evolution Computation, vol. 13, no. 5, pp. 945-958, 2009.

[24] S. Baluja, "Population-based incremental learning: a method for integrating genetic search based function optimization and competitive learning," Technical Report CMU_CS_95_ 163, Carnegie Mellon University, Pittsburgh, PA, USA, 1994.

[25] S. Mirjalili, S. M. Mirjalili, and A. Lewis, "Grey wolf optimizer," Advances in Engineering Software, vol. 69, pp. 46-61, 2014.

[26] S. Mirjalili, "SCA: a sine cosine algorithm for solving optimization problems," Knowledge-Based Systems, vol. 96, pp. 120-133, 2016

[27] K. Wansaseub, N. Pholdee, and S. Bureerat, "Optimal Ushaped baffle square-duct heat exchanger through surrogateassisted self-adaptive differential evolution with neighborhood search and weighted exploitation-exploration," Applied Thermal Engineering, vol. 118, pp. 455-463, 2017.

[28] S. Bureerat and N. Pholdee, "Adaptive sine cosine algorithm integrated with differential evolution for structural damage detection," in Proceedings of Computational Science and Its Applications (ICCSA 2017), vol. 10404, pp. 71-86, Trieste, Italy, July 2017.

[29] K. Wichapong, S. Bureerat, and N. Pholdee, "Solving inverse kinematics of robot manipulators by means of meta-heuristic optimisation," IOP Conference Series: Materials Science and Engineering, vol. 370, no. 1, article 012056, 2018.

[30] S. Bureerat and N. Pholdee, "Inverse problem based differential evolution for efficient structural health monitoring of trusses," Applied Soft Computing Journal, vol. 66, pp. 462-472, 2018.

[31] S. Sleesongsom and S. Bureerat, "Topology optimisation using MPBILs and multi-grid ground element," Applied Sciences, vol. 8 , no. 2 , p. $271,2018$. 


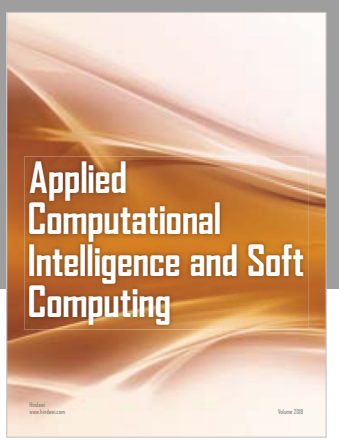

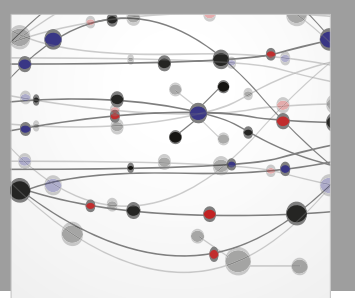

The Scientific World Journal
Submit your manuscripts at

Computing
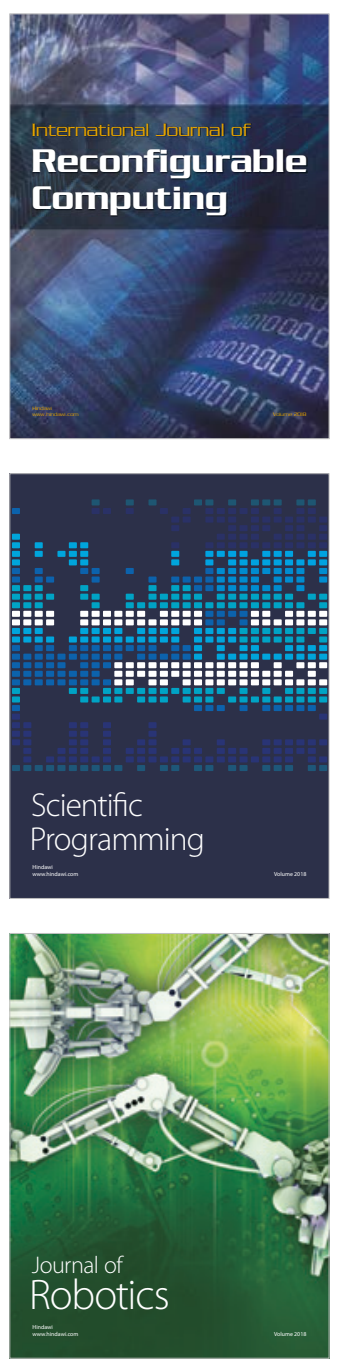



Human-Compute

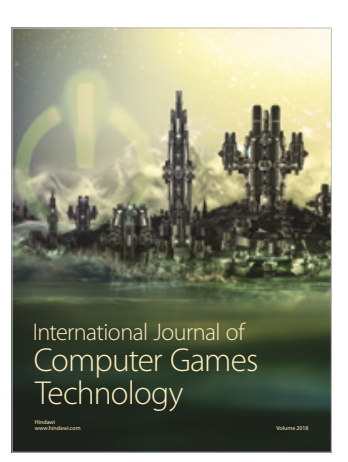

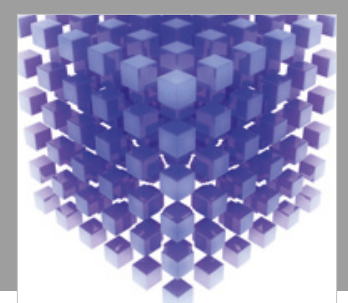

Mathematical Problems in Engineering

\section{Engincering}
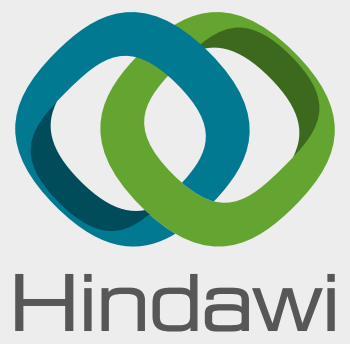

www.hindawi.com
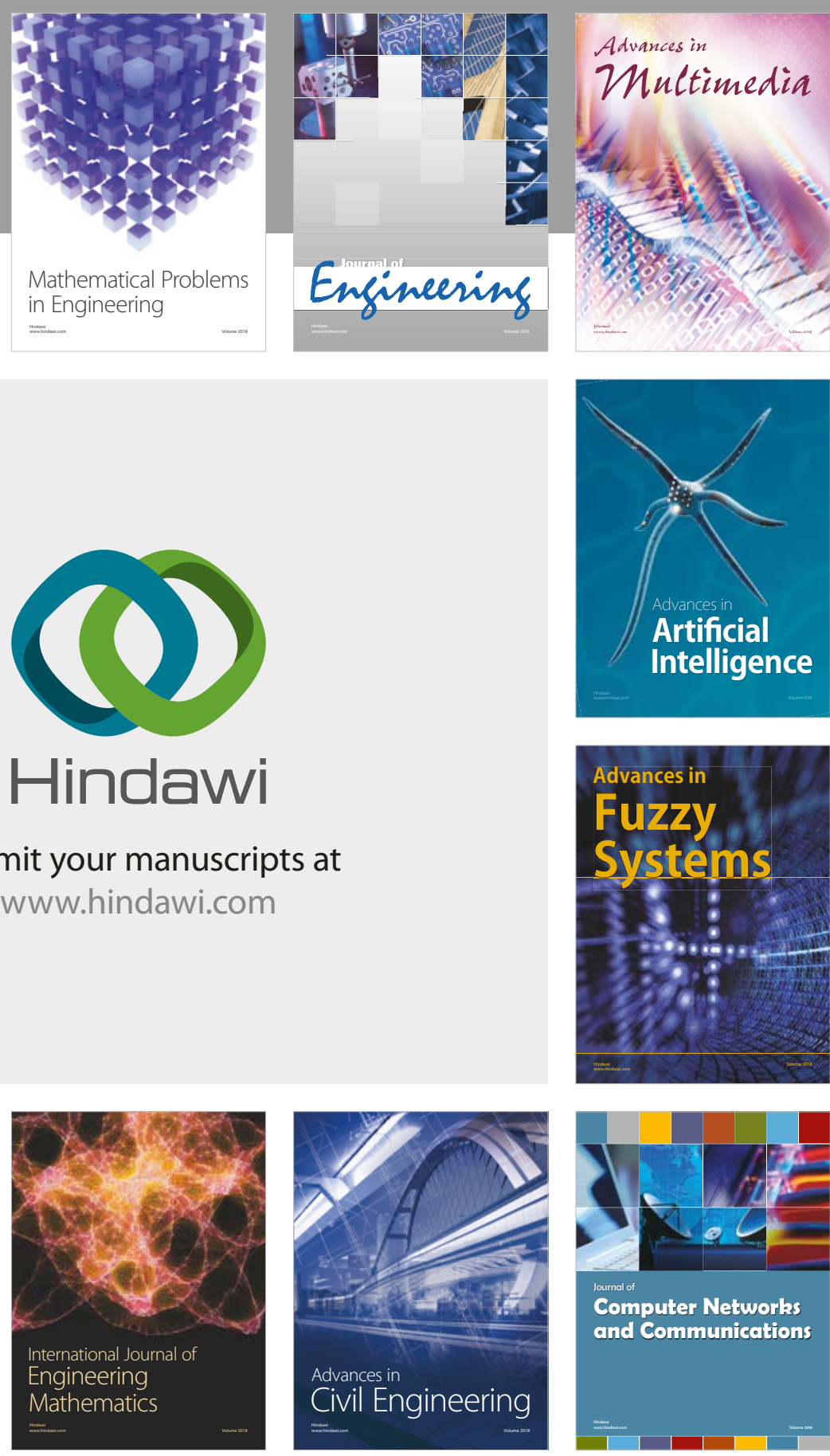

Computer Networks and Communications

Multimedia


Advances in

Modelling \&

Simulation

in Engineering

interaction

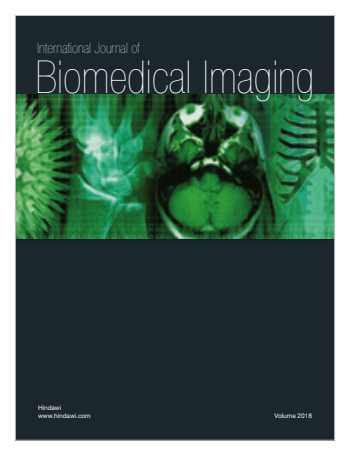

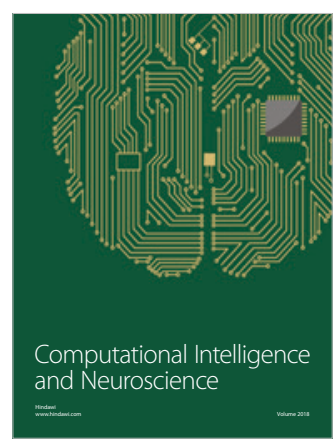

